\title{
Géomorphologie et gestion du patrimoine naturel. La mémoire de la Terre est notre mémoire.
}

\begin{abstract}
Résumé
Les autorités du canton de Fribourg (Suisse) ont décidé récemment de se doter d'un plan sectoriel des paysages et des sites. L'intégration des caractéristiques géomorphologiques dans ce plan d'aménagement a suscité une réflexion approfondie sur l'intérêt de cette discipline et sur les modalités de sa prise en compte dans le cadre de la gestion du milieu naturel.

Cet article présente un plan d'action répondant à ces préoccupations. II évoque les défis à surmonter dans les domaines suivants:

- recherche fondamentale:

- étudier les régions et phénomènes géomorphologiques (processus et formes) peu connus;

- analyser les relations relief - milieu naturel;

- étudier le rôle du relief dans la perception du paysage;

- recherche appliquée:

- collecter les données de base concernant les phénomènes géomorphologiques;

- apprécier la valeur des phénomènes géomorphologiques;

- cerner les menaces qui pèsent sur les phénomènes géomorphologiques;

- proposer des mesures de gestion;

- législation: intégrer la protection du patrimoine géomorphologique dans la législation;

- information: sensibiliser le public à la valeur du patrimoine géomorphologique.
\end{abstract}

Mots-clés: géomorphologie, gestion du patrimoine naturel, Fribourg (Suisse).

\section{Geomorphology and Management of Natural Heritage. \\ The Memory of the Earth is our Memory.}

\begin{abstract}
Recently, the government of the canton of Fribourg (Switzerland) has decided to establish a sector plan that includes directives specific to nature conservation: the Sector Plan for Landscapes and Sites. The integration of geomorphological characteristics in this land-use plan has prompted further reflection on geomorphology's contributions to this field, as well as on the modalities necessary for its integration in management of the natural environment.

This article presents a plan of action responding to these concerns and evokes challenges to be met in the following domains:

- fundamental research:

- study little known geomorphological regions and phenomena (processes and landforms);
\end{abstract}

- analyze the relationship between relief and natural environment:

- study the role the relief plays in landscape perception;

- applied research:

- collect basic data concerning geomorphological phenomena;

- assess the value of geomorphological phenomena;

- define the threats to geomorphological phenomena;

- propose adequate management measures for geomorphological phenomena;

- legislation: integrate the conservation of the geomorphological heritage into current legislation;

- information: increase public awareness of the value of the geomorphological heritage.

Keywords: geomorphology, management of natural heritage, Fribourg (Switzerland).

\section{Introduction}

\subsection{Le patrimoine naturel}

Selon DONADIEU (1986, p. 73), «a valeur de patrimoine ce qui doit être transmis à nos descendants dans le meilleur état possible... Il s'agit donc de ressources faiblement, difficilement ou non renouvelables qui exigent en premier lieu des mesures réglementaires de protection.» L'environnement naturel constitue le cadre de vie de tous les êtres vivants et de l'homme en particulier. De par sa complexité, sa dynamique et sa sensibilité, l'environnement naturel et son histoire représentent un patrimoine pour les sociétés humaines (MARTINI 1994).

La gestion du patrimoine naturel est une des tâches fondamentales de l'aménagement du territoire et de la protection de la nature. Malheureusement, le patrimoine naturel est le plus souvent appréhendé de façon restrictive comme n'englobant que la faune, la flore et les biotopes les plus remarquables, au mépris des conditions géologiques et géomorphologiques qui constituent pourtant le substrat de la vie.

Vincent Grandgirard, Géographe dipl., Université de Fribourg Institut de Géographie, Pérolles, $\mathrm{CH}-1700$ Fribourg. 


\subsection{Le patrimoine géologique et géomorphologique}

Le patrimoine géologique et géomorphologique est parfois intégré à la gestion du milieu naturel, notamment dans le cadre de la protection des géotopes, qui sont des «portions de la géosphère présentant une importance particulière pour la compréhension de l'histoire de la Terre» (GRANDGIRARD 1995). Ce patrimoine est la mémoire du passé de la Terre et une des clés pour la compréhension de son présent et de son futur. Toute forme d'aménagement doit tenir compte de la valeur intrinsèque et de la singularité de ce patrimoine (COLL. 1994, DALY et al. 1994). Cela est d'autant plus important que le patrimoine géo(morpho)logique est fini et non renouvelable.

Dans une optique plus utilitaire, la préservation de ce patrimoine se justifie pour les raisons suivantes (DALY et al. 1994, NATURE CONSERVANCY COUNCIL 1990; WILSON 1994):

- pour permettre le progrès des connaissances dans le domaine des sciences de la Terre et pour améliorer notre compréhension des processus par lesquels le milieu naturel évolue;

- pour disposer de sites intacts et exemplaires pouvant servir d'illustrations concrètes dans le cadre de la formation des spécialistes en sciences de la Terre. Pour ces derniers, le terrain représente en effet un laboratoire d'étude irremplaçable;

- pour fournir aux enseignants et à leurs élèves (à tous les niveaux du cursus scolaire) ainsi qu'à toutes les personnes intéressées (p. ex. les amoureux de la nature, les collectionneurs de fossiles ou de minéraux, les spéléologues) des sites accessibles et bien documentés leur permettant de se familiariser avec la Terre et son histoire;

- pour préserver la valeur esthétique et paysagère, l'importance écologique, l'intérêt culturel ou historique des sites géo(morpho)logiques. Par le cumul de ces qualités, ces sites constituent des points d'ancrage de l'identité collective.

Les éléments exposés ci-dessus démontrent que la conservation du patrimoine géo(morpho)logique sert à la fois les intérêts de la nature, ceux des spécialistes en sciences de la Terre ainsi que ceux de la population en général.

\subsection{La situation fribourgeoise}

Du fait de sa localisation entre le Plateau molassique et les Alpes (figure 1), le canton de Fribourg $\left(1670 \mathrm{~km}^{2}\right)$ est caractérisé par un environnement naturel très diversifié et très riche.

Le plan directeur cantonal - FR 87 (DIRECTION DES TRAVAUX PUBLICS 1987) est le principal instrument de coordination pour l'aménagement du territoire du canton de Fribourg. Selon ce dernier, la gestion des paysages et des sites est régie par l'inventaire des sites naturels (DIRECTION DES TRAVAUX PUBLICS 1978). Aujourd'hui, cet instrument est considéré comme insuffi-

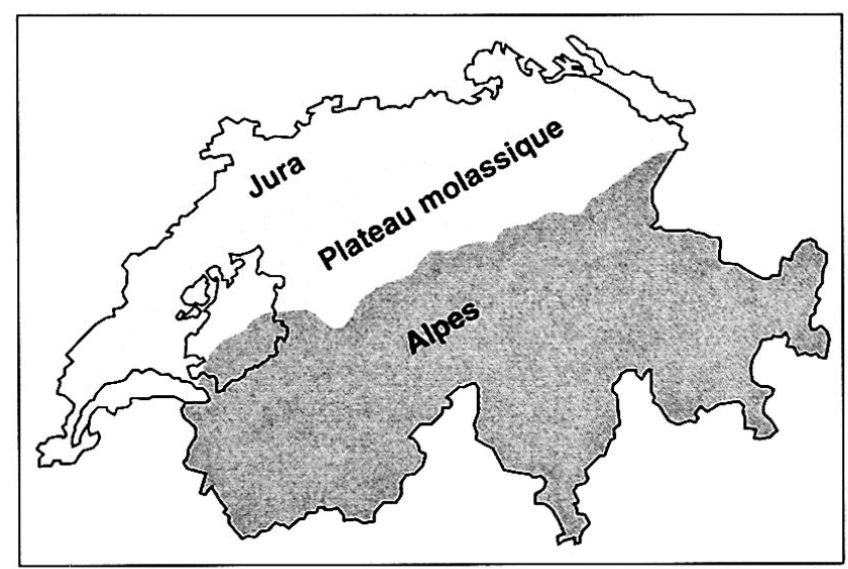

Fig. 1 Localisation du canton de Fribourg en Suisse.

sant et le plan directeur - FR 87 prévoit l'élaboration d'un plan sectoriel des paysages et des sites, destiné à le remplacer. Ce nouvel outil de gestion, actuellement en cours de réalisation (TEAM+ 1993 et 1995), intègre les composantes géologiques et géomorphologiques.

\section{La gestion du patrimoine géomorphologique}

Alors que certains pays européens, tels que l'Allemagne (GRUBE 1994, WIEDENBEIN 1994, AD-HOC-ARBEITSGRUPPE GEOTOPSCHUTZ 1996) et surtout la Grande-Bretagne (NATURE CONSERVANCY COUNCIL 1990, O'HALLORAN 1994, WILSON 1994), sont très avancés sur le plan de la conservation du patrimoine géo(morpho)logique, la Suisse est quelque peu à la traîne dans ce domaine (STRASSER et al. 1995; STÜRM 1994 a et 1994 b). En effet, malgré un système d'aménagement moderne et bien développé, les bases légales et les instruments de planification sont insuffisants et doivent être complétés.

Dans ce contexte peu favorable, plusieurs régions et cantons de Suisse ont fait œuvre de pionnier en se dotant d'outils de gestion du patrimoine géo(morpho)logique. Sur ce plan, le canton de Fribourg peut être qualifié de progressiste. En effet, dès le début de l'élaboration du plan sectoriel des paysages et des sites (TEAM+ 1993 et 1995), les responsables cantonaux de l'aménagement du territoire et de la protection de la nature se sont assurés l'aide de spécialistes (géomorphologues et géologues). Cette collaboration a donné lieu à un vaste projet de recherche appliquée qui est à l'origine de nombreuses propositions et réalisations novatrices.

La plupart de celles-ci sont présentées dans le présent article, qui constitue en quelque sorte un plan d'action pour la prise en compte de la géomorphologie dans le cadre de la gestion du patrimoine naturel. La figure 2 présente les multiples aspects de cette ambitieuse entreprise. Les éléments de ce plan d'action sont commentés et, lorsque cela est possible, illustrés par des expériences effectuées en Suisse et dans le canton de Fribourg. 


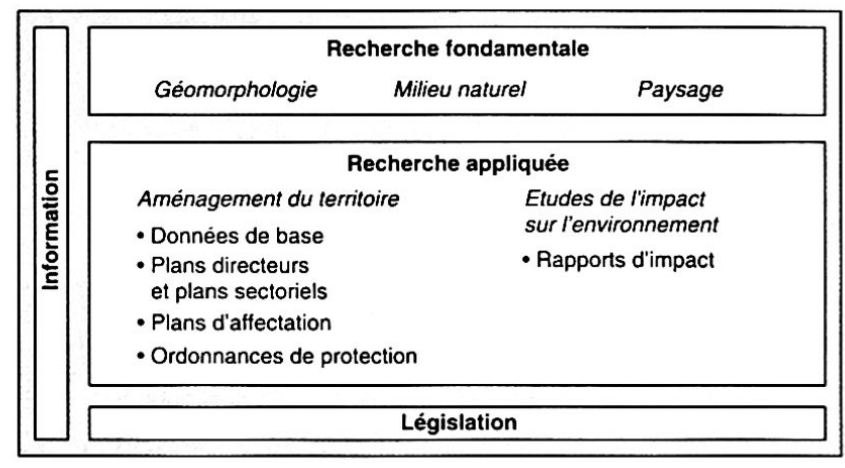

Fig. 2 Domaines concernés par la géomorphologie dans le cadre de la gestion du patrimoine naturel et du paysage.

\subsection{Recherche fondamentale}

La géomorphologie est une discipline essentielle pour l'étude et la gestion de l'environnement. Outre son utilité dans le cadre de la conservation du patrimoine naturel (chap. 1.), la géomorphologie livre des données essentielles pour la gestion des ressources (p. ex. les matériaux de construction, l'eau, l'énergie, le sol), pour la prévention des risques naturels (p. ex. les instabilités de terrain, les avalanches, les inondations), pour l'agriculture et la sylviculture (p. ex. l'irrigation, l'érosion des sols), pour l'étude des paysages, etc.

Afin d'accroître encore la pertinence et, par conséquent, l'intérêt des observations géomorphologiques, la recherche fondamentale doit être poursuivie et même intensifiée. Il convient d'encourager en particulier les recherches portant sur les régions et phénomènes peu connus ainsi que les recherches interdisciplinaires portant sur le milieu naturel ou sur le paysage (figure 2).

\subsubsection{Géomorphologie}

Dans le domaine strict de la géomorphologie, les efforts de la recherche fondamentale portent principalement sur trois aspects interdépendants: l'inventaire et la description des formes du relief, la compréhension des processus morphogéniques par lesquels ces formes évoluent et la reconstitution des étapes de l'évolution du relief.

La cartographie morpho-processuelle permet de représenter à la fois les formes du relief, les processus responsables de leur genèse et leur dynamique actuelle. Elle constitue un des moyens les plus performants et les plus utiles pour la transmission du savoir géomorphologique (STÜRM 1983, COOKE \& DOORNKAMP 1974).

Les progrès de la géomorphologie et la reconnaissance de son utilité reposent en grande partie sur la conduite de recherches approfondies portant sur des régions ou des phénomènes peu étudiés.

\subsubsection{Milieu naturel}

Les phénomènes géomorphologiques sont en étroite interdépendance avec de nombreuses composantes du mi- lieu naturel (composantes géologiques - lithologie et structure; hydro(géo)logiques - écoulements superficiels et souterrains; pédologiques - nature des sols et pédogenèse; écologiques - distribution de la végétation; climatiques; etc.). Du fait de ces interactions, la géomorphologie constitue un élément fondamental de description et de compréhension du milieu naturel (GRANDGIRARD 1994).

L'analyse isolée des multiples composantes du milieu naturel en fournit une image fragmentaire. En effet, le milieu naturel ne peut être pleinement appréhendé que par le biais d'études interdisciplinaires. L'étude des interactions entre la géomorphologie et les autres composantes du milieu naturel représente un défi pour les géomorphologues.

\subsubsection{Paysage}

Les formes du relief constituent une composante de base du paysage. En effet, le soubassement géomorphologique est le support de la couverture végétale et des éléments culturels (constructions, mais aussi mode d'occupation du sol et de mise en valeur des sites, etc.) qui forment, ensemble, le paysage (GRANDGIRARD 1994). Les contributions des géomorphologues aux études paysagères consistent le plus souvent en inventaires des formes ou combinaisons de formes qui apparaissent au sein d'un paysage (BAUMGARTNER 1982, DOLLINGER 1986, STUBER 1993). L'importance paysagère des éléments du relief n'est par contre que rarement ou sommairement appréciée (COOKE \& DOORNKAMP 1974). Cette dernière thématique constitue pourtant un vaste champ d'investigation pour les géomorphologues (encadré 1), qui pourraient développer de nouveaux instruments d'analyse basés sur l'exploitation de données numériques telles que les modèles numériques de terrain et l'imagerie satellitaire. Le rôle joué par le relief dans la perception des paysages et dans la formation de l'identité territoriale représente également une problématique de recherche interdisciplinaire peu explorée.

\subsection{Recherche appliquée}

Dans le cadre de cet exposé, nous nous limiterons à examiner les apports de la géomorphologie à la gestion du patrimoine naturel. Deux domaines d'activité sont principalement concernés: l'aménagement du territoire et les études de l'impact sur l'environnement (figure 2). Les bases légales régissant ces deux domaines ainsi que leurs implications pour la gestion du patrimoine géomorphologique sont discutées dans le chapitre 2.3.

En Suisse, dans le cadre de l'aménagement du territoire, de la protection de la nature et du paysage ainsi que de la protection de l'environnement, les quatre principaux instruments de gestion du milieu naturel et du paysage sont (figure 2):

- les plans directeurs et les plans sectoriels (niveau cantonal et communal): ils fixent les lignes directrices de l'aménagement du territoire, en fonction du dévelop- 


\section{Encadré 1 - L'importance paysagère des formes du relief}

L'évaluation de l'importance paysagère des éléments du relief est une opération délicate qui peut, si elle n'est pas effectuée selon une procédure rigoureuse, être très influencée par la subjectivité de l'observateur.

Selon notre conception, un paysage stricto sensu est une portion d'espace perçue par un spectateur donné, à un moment précis, depuis un emplacement défini à la surface de la Terre (Grandgirard, 1997). L'importance paysagère des constituants du paysage, et par conséquent des formes du relief, dépend essentiellement de leurs caractéristiques visuelles. Elle s'apprécie en fonction de leur visibilité et de leur rôle paysager (figure).

\section{Visibilité}

- Points de vue et distance d'observation

- Etendue et développement vertical

- Position (dominante/dominée)

- Contraste avec l'environnement (contraste de couleur en particulier)

\section{Rôles paysagers}

- Points d'appel et focalisation du regard

- Lignes de force du paysage

- Obstacles visuels et plans paysagers

- Contrastes avec l'environnement (en général)

La visibilité rend compte de la capacité d'un élément du paysage à être perçu par le sens de la vue. La visibilité dépend des caractéristiques suivantes:

- les points de vue et la distance d'observation: la visibilité d'un objet est d'autant plus grande que les points de vue sont nombreux et la distance d'observation importante;

- l'étendue et le développement vertical: un objet est d'autant plus visible que son étendue et son développement vertical sont grands;

- la position (dominante/dominée): la position dominante d'un objet par rapport à son environnement lui confère une visibilité accrue, même si son développement vertical est restreint;

- le contraste avec l'environnement (contraste de couleur en particulier): un objet qui, du fait de sa couleur, contraste avec son environnement est plus aisément repérable.

L'analyse des caractéristiques visuelles ne se réduit cependant pas à l'étude de la visibilité. Les constituants du paysage, et surtout les formes du relief, jouent en effet un rôle fondamental dans la structuration du paysage, auquel ils confèrent une certaine cohérence (ou absence de cohérence). Les logiques de l'organisation du paysage peuvent être appréhendées par l'étude des rôles paysagers des constituants du paysage, qui peuvent être:

- les points d'appel et la focalisation du regard: les points d'appel paysagers canalisent, orientent le regard; ils constituent souvent des repères forts;

- les lignes de force du paysage: elles correspondent aux principaux axes structurants du paysage. Par leur agencement, les lignes de forces horizontales, verticales ou obliques déterminent l'équilibre, l'harmonie du paysage;

- les obstacles visuels et les plans paysagers: le paysage est compris dans un continuum d'échelles allant de la grande échelle (zone des détails, avant-plan) à la petite échelle (perception des volumes, des formes générales, arrière-plan) en passant par l'échelle moyenne (le plan du paysage proprement dit). Par leur rôle d'obstacles visuels, les constituants du paysage contribuent à définir et à structurer les plans paysagers;

- les contrastes avec l'environnement (en général): les contrastes de forme, de couleur et de texture sont des éléments importants de diversification et de structuration du paysage. 
pement souhaité. Ces plans sont les principaux instruments de coordination des activités ayant des répercussions sur l'organisation du territoire.

- les plans d'affectation (niveau communal surtout): par la délimitation de zones (p. ex. zones à bâtir, zones agricoles, zones forestières ou zones protégées), ils définissent de façon détaillée le mode d'utilisation du sol;

- les ordonnances de protection (niveau national, cantonal et communal): elles règlent en détail la protection de sites remarquables;

- les études de l'impact sur l'environnement (pour certains projets d'installations): les rapports d'impact fournissent toutes les indications relatives aux incidences sur l'environnement des installations projetées.

Ces instruments de gestion pourraient aisément intégrer des dispositions particulières concernant les phénomènes géomorphologiques. Malheureusement, les planificateurs ne possèdent généralement ni les données de base ni les compétences qui leur permettraient d'en tenir compte.

Dans certains cas, encore trop rares, les géomorphologues collaborent avec les gestionnaires du milieu naturel et du paysage. Les multiples attentes de ces derniers à leur égard se rangent en quatre catégories:

- collecte des données de base concernant les phénomènes géomorphologiques;

- aide à l'appréciation de la valeur des phénomènes géomorphologiques;

- aide à l'appréciation des menaces qui pèsent sur les phénomènes géomorphologiques;

- proposition de mesures de gestion adéquates pour les phénomènes géomorphologiques.

\subsubsection{Géomorphologie, plans directeurs \\ et plans sectoriels}

Dans le canton de Fribourg, le plan sectoriel des paysages et des sites (TEAM+ 1993 et 1995) se compose de trois volets distincts, présentés sous forme de cartes:

- une carte de l'état bioécologique et du degré de sollicitation des entités géographiques;

- une carte des sites naturels, des sites culturels et des géotopes;

- une carte des paysages d'intérêt cantonal.

\section{- La carte de l'état bio-écologique et du degré de sollicitation des entités géographiques}

L'état bioécologique des entités géographiques est apprécié en fonction de l'importance, du nombre, de la qualité, de la diversité et de la proximité des objets naturels et semi-naturels qui déterminent le fonctionnement du paysage en tant que réseau-habitat. La prise en compte du degré de sollicitation humaine pour chaque entité permet d'effectuer un diagnostic global de l'état du paysage dont découlent des objectifs particuliers (TEAM+ 1995 et 1996).
Pour aider à la délimitation des entités géographiques, définies comme des espaces homogènes, une étude géomorphologique portant sur l'ensemble du territoire cantonal a été réalisée (GRANDGIRARD 1994, GRANDGIRARD \& MONBARON 1995). Cet "aperçu géomorphologique» consiste essentiellement en une partition du territoire cantonal, fondée sur des critères géomorphologiques. Les limites des entités définies, qui correspondent souvent à des transitions géologiques, hydrologiques, pédologiques, écologiques ou même climatiques, ont fréquemment été associées aux frontières des entités géographiques.

\section{- La carte des sites naturels, des sites culturels et des géotopes}

Le plan sectoriel des paysages et des sites procède à la sélection de sites remarquables. Ces derniers sont considérés comme présentant un intérêt cantonal et leur gestion s'appuie sur un catalogue d'objectifs particuliers (TEAM+ 1995).

L'inventaire des géotopes géomorphologiques du canton de Fribourg (GRANDGIRARD 1996 et à paraître) recense plus de 300 objets géomorphologiques de valeur, représentatifs de la diversité des reliefs fribourgeois et méritant une attention particulière. La sélection de ces géotopes repose sur l'emploi d'une méthode originale permettant d'apprécier la valeur scientifique des objets géomorphologiques (encadré 2).

Afin de définir des mesures de gestion pour ces géotopes, il convient de procéder à l'évaluation de leur importance paysagère (encadré 1) et à l'appréciation des menaces qui pèsent sur ces géotopes. En fonction de ce dernier aspect, nous distinguons:

- les géotopes menacés dans leur intégrité ou leur activité morphogénique;

- les géotopes menacés en tant qu'éléments caractéristiques ou prégnants du paysage;

- les autres géotopes, qui ne sont pas directement menacés.

Les atteintes aux formes du relief sont en général irréversibles, ces dernières ne pouvant être reconstituées ou compensées. De ce fait, les mesures de gestion des géotopes géomorphologiques sont essentiellement des mesures de protection (p. ex. création d'une réserve naturelle, restriction de l'utilisation) ou de mise en valeur (p. ex. création d'un sentier didactique, information, amélioration de l'accessibilité).

A l'instar des expériences menées en Grande-Bretagne (NATURE CONSERVANCY COUNCIL 1990, O'HALLORAN 1994, WILSON 1994), la gestion de ces géotopes pourrait être confiée à des groupes d'intérêt locaux ou régionaux, concernés par leur conservation (sociétés scientifiques, associations de protection de la nature, musées, écoles, universités, planificateurs, autorités, propriétaires, etc.).

\section{- La carte des paysages d'intérêt cantonal}

Du fait de leurs caractéristiques d'ensemble (du point de vue naturel, culturel et géologique-géomorphologique), certaines portions du territoire constituent des paysages 


\section{Encadré 2 - L'évaluation des objets géomorphologiques}

Les critères utilisés pour l'évaluation des objets géomorphologiques doivent permettre d'apprécier leur valeur scientifique, du point de vue de la géomorphologie.

En fonction de leur importance dans le processus d'évaluation, nous distinguons deux types de critères: les facteurs et les indicateurs (figure). Les facteurs sont les critères fondamentaux. La valeur d'un objet géomorphologique correspond en effet à la combinaison des résultats obtenus lors de l'appréciation de chaque facteur. Les indicateurs sont des critères secondaires, à prendre en considération lors de l'évaluation des facteurs. Un indicateur donné peut servir à l'appréciation de plusieurs facteurs.

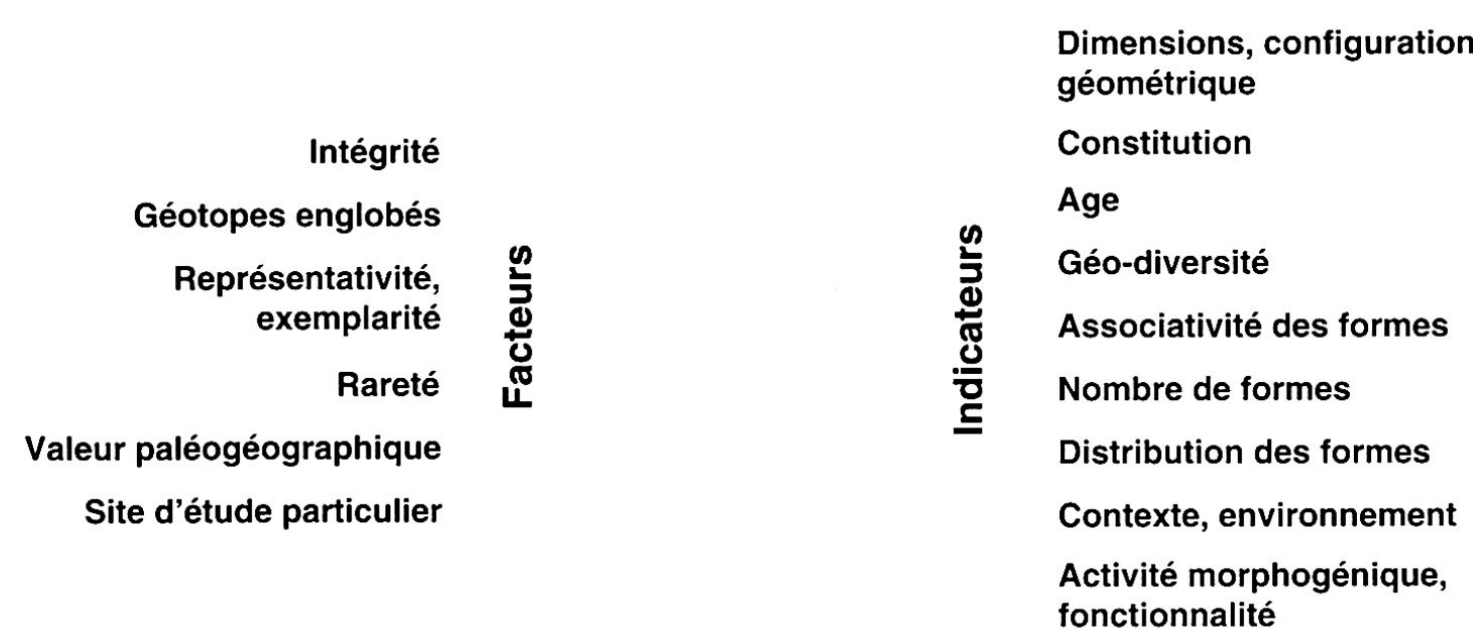

Seuls les facteurs, qui sont les critères prépondérants, font l'objet d'un commentaire.

- Intégrité: correspond au degré de préservation des caractéristiques originelles de l'objet considéré. Elle est appréciée essentiellement en fonction de l'importance des sollicitations humaines.

- Géotopes englobés: les géotopes géomorphologiques englobant d'autres géotopes sont valorisés, de façon comparable aux biotopes qui recèlent des espèces rares ou menacées.

- Représentativité, exemplarité: ce facteur renvoie à la valeur didactique et à la lisibilité des phénomènes (formes et processus) observés. La représentativité des objets géomorphologiques s'apprécie par comparaison avec d'autres objets du même type, d'après leur aptitude à rendre compte de l'activité passée ou actuelle du processus responsable de leur genèse et, pour les objets les plus complexes, en fonction de leur capacité à illustrer les caractéristiques d'une «région géomorphologique», cette dernière étant conditionnée par les conditions lithologiques, structurales et climatiques approximativement uniformes qui y règnent.

- Rareté: la rareté s'évalue en fonction d'un espace de référence. La rareté que l'on s'efforce d'évaluer est à la fois celle du type d'objet géomorphologique considéré et celle de l'objet étudié, du fait de ses particularités.

- Valeur paléogéographique: certains objets géomorphologiques constituent des témoins irremplaçables de l'histoire de la Terre. Leur valeur paléogéographique dépend des informations qu'ils sont susceptibles de livrer compte tenu de leur configuration géométrique, de leur constitution, de la distribution des formes qu'ils recèlent, de leur environnement et de leur localisation, de leur âge, de leur activité, etc.

- Site d'étude particulier: la valeur d'un objet géomorphologique en tant que site d'étude est liée aux connaissances accumulées ou à celles susceptibles d'être fournies par des recherches en cours ou futures. 
d'intérêt cantonal. Le plan sectoriel des paysages et des sites vise le respect des différentes composantes du paysage et l'amélioration de leur valeur d'ensemble (TEAM+ 1995).

En vertu de cette conception, l'aperçu géomorphologique (GRANDGIRARD 1994, GRANDGIRARD \& MONBARON 1995 ) et l'inventaire des géotopes géomorphologiques (GRANDGIRARD 1996 et à paraître) constituent des données de base essentielles pour la détermination des paysages d'intérêt cantonal. Les menaces et les mesures de gestion se rapportant aux phénomènes géomorphologiques sont identiques à celles présentées ci-dessus pour les géotopes. Au sein des paysages d'intérêt cantonal, elles s'appliquent également aux éléments de moindre importance, qui contribuent à la valeur d'ensemble et qui ne sons pas protégés par des dispositions particulières.

Le développement et l'application de méthodes numériques permettant l'analyse approfondie des caractéristiques visuelles du paysage (visibilité et rôles paysagers) constitueraient une aide inestimable pour la détermination des menaces qui pèsent sur les paysages d'intérêt cantonal et pour la définition de mesures de gestion appropriées.

\subsubsection{Géomorphologie et plans d'affectation}

Les actions qu'entraîne la mise en application du plan sectoriel des paysages et des sites ne sont pas exclusivement l'affaire du canton. En effet, les communes doivent prendre connaissance des objectifs et principes édictés par le plan sectoriel et en tenir compte lors des révisions ou des adaptations de leur plan d'affectation (TEAM+ 1995).

Par ignorance ou par manque d'information, les caractéristiques géomorphologiques risquent cependant de ne pas être considérées à leur juste valeur lors de ces modifications. En vue de démontrer l'importance qu'il y aurait à tenir compte de ces caractéristiques et afin de fournir des exemples concrets de mise en pratique, il serait utile de réaliser, pour quelques communes-tests, un plan d'affectation modifié intégrant les données suivantes:

- patrimoine géomorphologique:

- étude géomorphologique du territoire communal;

- inventaire des objets géomorphologiques, évaluation (valeur scientifique, encadré 2) et classification de ces objets (valeur locale, cantonale, nationale);

- appréciation des menaces qui pèsent sur ces objets, tant du point de vue de leur intégrité que de leur fonctionnement;

- proposition de mesures de protection et de mise en valeur;

- patrimoine paysager (caractéristiques visuelles du paysage):

- étude de la visibilité (réalisation d'une carte de la soumission à la vue) et du rôle des reliefs dans la structure du paysage;
- évaluation de l'importance paysagère des objets géomorphologiques (encadré 1);

- appréciation des menaces qui pèsent sur la visibilité et les rôles paysagers des objets géomorphologiques;

- proposition de mesures de protection et de mise en valeur.

\subsubsection{Géomorphologie et ordonnances de protection}

En Suisse, des ordonnances de protection peuvent être édictées à tous les niveaux de planification (national, cantonal et communal). Parmi les nombreuses ordonnances de ce type existant au niveau national (p. ex. inventaire des paysages, sites et monuments naturels; protection des hauts-marais et des marais de transition d'importance nationale; protection des zones alluviales d'importance nationale), aucune ne se rapporte directement au patrimoine géomorphologique.

Le Groupe de travail pour la protection des géotopes en Suisse, qui rassemble des spécialistes des différentes disciplines des sciences de la Terre, prépare actuellement un inventaire des géotopes d'importance nationale. Complété par un guide sur la protection des géotopes, un tel document pourrait constituer un premier pas en direction de la réalisation d'une «Ordonnance sur la protection des géotopes d'importance nationale».

\subsubsection{Géomorphologie et études de l'impact sur l'environnement (EIE)}

En guise de remarque préliminaire, il est utile de rappeler qu'une EIE doit permettre de déterminer si un projet d'installation correspond aux prescriptions fédérales sur la protection de l'environnement au sens large (y compris les dispositions concernant la protection de la nature et du paysage ou l'aménagement du territoire).

La géomorphologie est le plus souvent ignorée ou traitée subsidiairement dans les EIE. Les phénomènes géomorphologiques semblent pris en compte de façon implicite, comme s'il allait de soi que toute biocénose ou infrastructure construite repose sur un substratum, ce dernier étant perçu comme quasi intangible, voire totalement indifférent à ce contact (MONBARON 1993). Récemment, l'Office Fédéral de l'Environnement, des Forêts et du Paysage (OFEFP 1991) a émis des recommandations mentionnant explicitement la géomorphologie comme une des disciplines-actrices de l'EIE. Plusieurs auteurs ont d'ailleurs déjà contribué à cerner l'utilité de la géomorphologie dans le cadre d'études de ce type (KIENHOLZ et al. 1988, MONBARON 1993, GSTEIGER 1995).

L'EIE est basée sur un rapport, le rapport d'impact (figure 2). Ce dernier décrit d'abord l'état initial de l'environnement aux abords de l'installation prévue. Dans un second temps, il rend compte des atteintes que porterait cette installation à l'environnement. Enfin, il définit les mesures à prendre pour remédier à ces atteintes. Du fait de la structure du rapport d'impact, une démarche d'analyse particulière doit être suivie. La 
démarche présentée ci-dessous a été développée au sein de notre groupe de recherches dans le cadre de l'EIE pour un projet autoroutier.

La prise en compte des caractéristiques géomorphologiques est requise lors de l'appréciation des impacts sur les géotopes, sur le paysage dans son ensemble et sur les formes du relief, considérées comme constituants du paysage.

Pour les impacts sur les géotopes géomorphologiques, il convient:

- d'apprécier l'état initial:

- de recenser les objets géomorphologiques touchés par le projet;

- de les étudier (localisation, conformation, distribution, contexte, morphogenèse et activité actuelle, etc.);

- d'en estimer la valeur scientifique (encadré 2);

- d'évaluer les impacts causés par le projet sur les objets géomorphologiques: atteinte à leur intégrité (p. ex. destruction ou remblaiement) et/ou à leur activité morphogénique (artificialisation);

- de proposer des mesures de gestion supplémentaires (surtout protection et mise en valeur).

Pour les impacts sur le paysage dans son ensemble, il convient:

- d'apprécier l'état initial: réalisation d'une carte de la soumission à la vue et analyse du rôle des reliefs dans la structure du paysage;

- d'évaluer les impacts causés par le projet: réalisation d'une carte de l'aire de visibilité (surface d'où l'installation serait vue);

- de proposer des mesures favorisant l'intégration paysagère de l'installation (p. ex. localisation dans des espaces masqués).

Pour les impacts sur les formes du relief, considérées comme éléments du paysage, il convient:

- d'apprécier l'état initial:

- de recenser les formes du relief touchées par le projet;

- d'évaluer leur importance paysagère (encadré 1);

- d'évaluer les impacts paysagers causés par le projet sur les formes du relief;

- de proposer des mesures de gestion supplémentaires (protection et mise en valeur ainsi que, lorsque c'est possible, compensation pour les impacts paysagers).

\subsection{Législation}

\section{- Au niveau national et international}

En Suisse, les bases légales de l'aménagement du territoire, de la protection de la nature et de la protection de l'environnement ne mentionnent jamais explicitement le patrimoine géo(morpho)logique. Sans l'encourager, elles autorisent tout de même la protection de ce patrimoine, comme en témoignent les articles suivants (la liste n'est pas exhaustive):

- loi fédérale sur la protection de la nature et du paysage (LPN) du ler juillet 1966, article premier, a:
«... la présente loi a pour but: de ménager... les curiosités naturelles... du pays»;

- loi fédérale sur l'aménagement du territoire (LAT) du 22 juin 1979, art. 3, al. 2, d: «Le paysage doit être préservé. Il convient notamment: de conserver les sites naturels....»;

- LAT, art. 17, al. 1, a: «Les zones à protéger comprennent: les paysages... d'un grand intérêt pour les sciences naturelles...».

En regard des prescriptions concernant les biotopes, les bases légales assurant la protection du patrimoine géo(morpho)logique sont très lacunaires. Le Groupe de travail pour la protection des géotopes en Suisse fait des propositions pour que les géotopes soient considérés, dans les textes légaux et dans les directives de la Confédération, au même titre que les biotopes.

Selon STÜRM (1994 a et 1994 b), une convention internationale relative à la protection du patrimoine géo(morpho)logique, analogue à la Convention relative à la conservation de la vie sauvage et du milieu naturel de l'Europe (Convention de Berne), pourrait donner l'impulsion initiale et soutenir l'évolution souhaitée dans les différents états signataires.

\section{- Dans le canton de Fribourg}

La loi sur l'aménagement du territoire et les constructions (LATeC) du 9 mai 1983 est la seule base légale incluant des dispositions permettant la protection du patrimoine géo(morpho)logique. Ainsi, la loi «tend à assurer la sauvegarde des sites... dignes d'intérêt» (article premier, al. 2, a); elle stipule que «les études de base et les plans sectoriels concernent notamment: ...les territoires qui méritent une protection particulière du point de vue des sites naturels...» (art. 16, al. 2, c) et que «les zones de protection de la nature et du paysage comprennent: ...les paysages... d'un grand intérêt pour les sciences naturelles...» (art. 61, al. 1, b).

Une loi cantonale sur la protection de la nature et du paysage, qui considérerait les géotopes au même titre que les biotopes, pourrait assurer la protection du patrimoine géo(morpho)logique. Une telle loi fait malheureusement encore défaut dans le canton de Fribourg.

\subsection{Information}

L'efficacité de la gestion du patrimoine géo(morpho)logique dépend de la prise de conscience, par ses utilisateurs (chap. 1.2) et par les personnes impliquées dans sa protection, de la valeur de ce patrimoine. Les personnes impliquées peuvent être rangées en plusieurs catégories.

Nous distinguons:

- les scientifiques œuvrant dans le domaine des sciences de la Terre (chercheurs, enseignants, étudiants, sociétés scientifiques);

- les autres naturalistes (biologistes, écologues, pédologues, etc.);

- les aménagistes et les protecteurs de la nature (associations de protection de nature);

- les autorités à tous les niveaux décisionnels; 
- les propriétaires fonciers et les exploitants de géotopes (p. ex. exploitation de matériaux de construction ou utilisation de sites à des fins touristiques);

- les personnes intéressées aux sciences de la Terre et les musées;

- les écoles (enseignants et élèves);

- le grand public.

Afin de promouvoir la gestion du patrimoine géo(morpho)logique, les scientifiques concernés (surtout les géologues et les géomorphologues) doivent être animés d'un souci permanent d'information. La nature des informations à transmettre, le langage et les médias utilisés dépendent dans une large mesure du public-cible.

\section{- Les scientifiques}

\section{cuvrant dans le domaine des sciences de la Terre}

Principaux «utilisateurs» du patrimoine géo(morpho)logique, les scientifiques œuvrant dans le domaine des sciences de la Terre sont en général sensibles à sa valeur. Les aspects méthodologiques de la protection des géotopes (p. ex. l'appréciation de la valeur scientifique des phénomènes géomorphologiques) et des exemples de réalisations concrètes ( $\mathrm{p}$. ex. mise sous protection d'un site remarquable) peuvent leur être communiqués par le biais de publications dans des revues spécialisées ou d'exposés lors de colloques scientifiques. Informés, ces scientifiques se feront certainement avocats de la cause des géotopes.

\section{- Les autres naturalistes, les aménagistes et les protecteurs de la nature}

Il est essentiel que ces interlocuteurs soient sensibilisés à l'importance des sciences de la Terre pour la gestion du milieu naturel. La diffusion des résultats d'études interdisciplinaires impliquant des géologues ou des géomorphologues peut favoriser cette prise de conscience. La présentation des aspects méthodologiques de la protection des géotopes et leur illustration par des réalisations exemplaires pourront encourager l'intégration de cette dimension dans les pratiques de gestion du milieu naturel et du paysage.

Ces interlocuteurs peuvent être interpellés par le biais de communications dans des revues spécialisées ou lors de colloques. En outre, il est indispensable que les données de base existantes, telles que les inventaires de géotopes, soient mises à leur disposition. L'offre de cours de formation continue portant sur les aspects pratiques de la protection des géotopes (bases légales, valeur scientifique et paysagère, menaces, mesures de gestion, etc.) leur permettrait d'acquérir de nouvelles compétences. De tels cours pourraient également intéresser les spécialistes des sciences de la Terre, les autorités, ainsi que d'autres personnes impliquées.

\section{- Le grand public (y compris les personnes}

intéressées aux sciences de la Terre, les autorités, les propriétaires fonciers et les exploitants de géotopes) Le patrimoine géo(morpho)logique ne doit pas être la seule propriété du monde scientifique et des planifi- cateurs. Sa protection ne sera assurée que lorsque la population se sera approprié ce patrimoine et en sera devenu le véritable gardien (MARTINI 1994).

Les scientifiques concernés doivent faire une véritable promotion des sciences de la Terre auprès du grand public. Cette promotion doit porter d'une part sur les connaissances de base des sciences de la Terre et, d'autre part, sur les sites constituant le patrimoine géo(morpho)logique (SALVAN 1994).

Pour mener à bien cette tâche, géologues et géomorphologues doivent consentir à d'importants efforts de vulgarisation et utiliser tous les vecteurs d'informations disponibles. L'objet et l'utilité des sciences de la Terre doivent être rappelés aussi fréquemment que possible et en particulier lors d'événements exceptionnels (tels que crues, glissements de terrain, séismes, etc.). Les médias traditionnels (journaux, revues de grande diffusion, radio, télévision) doivent être utilisés de préférence. Certains géotopes doivent être aménagés de façon à ce que le grand public puisse appréhender sans peine ce patrimoine. En collaboration avec les musées, des expositions, des conférences et des visites guidées peuvent être organisées, des sentiers didactiques peuvent être réalisés, des brochures d'information peuvent être distribuées, etc.

La mise en valeur des géotopes peut en outre contribuer au développement touristique de régions économiquement défavorisées (notamment les régions de montagne). En effet, la visite de sites naturels exceptionnels représente souvent pour les touristes une occasion de se promener et de se distraire tout en se cultivant.

\section{- Les enseignants et leurs élèves}

Les enseignants constituent un public particulier. Ils peuvent en effet seconder les scientifiques dans leurs tâches de sensibilisation auprès du grand public. Il serait ainsi intéressant de mettre sur pied des cours de formation continue portant sur les thèmes des sciences de la Terre, des géotopes et de leur gestion. Ces cours pourraient être complétés par des excursions sur le terrain, susceptibles d'être proposées ultérieurement aux élèves. La mise à disposition de documentation vulgarisée concernant des sites proches des établissements scolaires encouragerait les enseignants à intégrer ces thèmes dans leurs leçons.

\section{Conclusion}

Selon la Déclaration internationale des droits de la mémoire de la Terre (COLL. 1994): «... La Terre nous porte. Nous sommes liés à la Terre et la Terre est lien entre chacun de nous. ... Notre histoire et l'histoire de la Terre sont intimement liées. Ses origines sont nos origines. Son histoire est notre histoire et son futur sera notre futur. Le visage de la Terre, sa forme, sont l'environnement de l'Homme. Cet environnement est différent de celui d'hier et différent de celui de demain. L'Homme est l'un des moments de la Terre; il n'est pas finalité, il est passage. ... la Terre conserve la mémoire 
du passé... une mémoire inscrite dans les profondeurs et sur la surface, dans les roches, les fossiles et les paysages, une mémoire qui peut être lue et traduite. ... Le passé de la Terre n'est pas moins important que le passé de l'Homme. Il est temps que l'Homme apprenne à protéger et, en protégeant, apprenne à connaître le passé de la Terre, cette mémoire d'avant la mémoire de l'Homme qui est un nouveau patrimoine: le patrimoine géologique. Le patrimoine géologique est le bien commun de l'Homme et de la Terre. Chaque Homme, chaque gouvernement n'est que le dépositaire de ce patrimoine. Chacun doit comprendre que la moindre déprédation est une mutilation, une destruction, une perte irrémédiable. ...»

Dans cette perspective, œuvrer pour la protection du patrimoine géologique et géomorphologique, c'est élargir notre compréhension de la nature afin d'établir une nouvelle relation entre l'Homme et la Terre. La tâche est énorme, mais la valeur de ce patrimoine et les enjeux liés à sa préservation méritent qu'on s'y attèle sans réserve.

\section{Bibliographie}

AD-HOC-ARBEITSGRUPPE GEOTOPSCHUTZ (1996): Geotopschutz in Deutschland. Leitfaden der Geologischen Dienste der Länder der Bundesrepublik Deutschland. Bonn-Bad Godesberg, Bundesamt für Naturschutz.

BAUMGARTNER, R. (1982): Einige Überlegungen zur Anwendung geomorphologischer Methoden für Praxis- oder landschaftsorientierte Untersuchungen. Dans: Materialien zur Physiogeographie, 4, 41-45.

COLL. (1994): Déclaration internationale des droits de la mémoire de la Terre. Dans: Mém. Soc. géol. France, N. S., 165. COOKE, R. U. \& DOORNKAMP, J. C. (1974): Geomorphology in Environmental Management. An Introduction. London, Clarendon Press.

DALY, D., ERIKSTAD, L. \& STEVENS, C. (1994): Fundamentals in earth science conservation. Dans: Mém. Soc. géol. France, N.S., 165, 209-212.

DIRECTION DES TRAVAUX PUBLICS (1978): Inventaire des sites naturels du canton de Fribourg. Fribourg, CCPNP et OCAT.

DIRECTION DES TRAVAUX PUBLICS (1987): Plan directeur cantonal - FR 87. Fribourg, OCAT.

DOLLINGER, F. (1986): Zum Problem der Bewertung von Naturlandschaften. Ein Plädoyer für eine geomorphologische Naturschutzarbeit. Dans: Natur und Land, 1, 15-20.

DONADIEU, P. (1986): Paysages et aménagement de I'espace. Dans: INRAP: Lectures du paysage. Paris, Foucher, 63-77.

GRANDGIRARD, V. (1994): Aperçu géomorphologique du canton de Fribourg. Fribourg, GReG, Institut de Géographie de l'Université de Fribourg.

GRANDGIRARD, V. (1995): Méthode pour la réalisation d'un inventaire de géotopes géomorphologiques. Dans: Ukpik, Cahiers de l'Institut de Géographie, Fribourg, 10, 121-137. GRANDGIRARD, V. (1996): Gestion du patrimoine naturel, l'inventaire des géotopes géomorphologiques du canton de Fribourg. Dans: Ukpik, Rapports de recherche, Institut de Géographie, Fribourg, 8, 181-195.

GRANDGIRARD, V. (1997): Du paysage au lieu de mémoire. Dans: Annales fribourgeoises, Tomes LXI et LXII, 41-48.
GRANDGIRARD, V. (à paraître): An inventory of geomorphological geotopes in the canton of Fribourg (Switzerland). Dans: Memorie descrittive della Carta geologica d'Italia, Acts of the II International Symposium on the Conservation of our Geological Heritage, Rome, May 20-21-22, 1996.

GRANDGIRARD, V. \& MONBARON, M. (1995): Aperçu géomorphologique du canton de Fribourg. Dans: Regio Basilensis, 36/2, 209-218.

GRUBE, A. (1994): Earth science conservation in Germany: an outline. Dans: Mém. Soc. géol. France, N. S., 165, 27-32. GSTEIGER, P. (1995): Die Bewertung von Oberflächenformen und reliefgestaltenden Prozessen im Rahmen einer Kraftwerks-UVP. Dans: Regio Basilensis, 36/2, 153-164.

KIENHOLZ, H., et al. (1988): Zur Beurteilung geomorphologischer Aspekte im Rahmen von Umweltverträglichkeitsprüfungen. Dans: Z. Geomorph. N. F., Suppl.-Bd. 70, 187-203. MARTINI, G. (1994): Bilan général de la protection du patrimoine géologique en France. Dans: Mém. Soc. géol. France, N. S., 165, 111-118.

MONBARON, M. (1993): La géomorphologie, élément indispensable dans toute étude d'impact sur l'environnement. Dans: Ukpik, Cahiers de I'Institut de Géographie, Fribourg, 9, 113-130.

NATURE CONSERVANCY COUNCIL (1990): Earth science conservation in Great Britain. A strategy. Peterborough, Nature Conservancy Council.

OFEFP (1991): Protection de la nature et du paysage lors de l'élaboration de rapports d'impact. Informations concernant l'étude de l'impact sur l'environnement, 4, Berne.

O'HALLORAN, D. (1994): Earth science conservation in Great Britain. The national picture. Dans: Mém. Soc. géol. France, N. S., 165, 159-161.

SALVAN, H. M. (1994): Un problème d'actualité: la sauvegarde du patrimoine géologique. Quelques réflexions. Dans: Mém. Soc. géol. France, N. S., 165, 229-230.

STRASSER, A., et al. (1995): Géotopes et protection des objets géologiques en Suisse: un rapport stratégique. Fribourg, Groupe de travail pour la protection des géotopes en Suisse.

STUBER, A. (1993): La géomorphologie dans les domaines de la protection de la nature et du paysage. Exemples d'applications cartographiques. Dans: Travaux et recherches, Institut de Géographie, Université de Lausanne, 9, 45-51.

STÜRM, B. (1983): Anwendungsmöglichkeiten und Anforderungen an geomorphologische Karten seitens der Raumplanung. Dans: Materialien zur Physiogeographie, 5, 29-32.

STÜRM, B. (1994 a): The geotope concept: geological nature conservation by town and country planning. Dans: O'HALLORAN, D., et al. (EDS): Geological and Landscape Conservation. Proceedings of the Malvern International Conference 1993. London, Geological Society, 27-31.

STÜRM, B. (1994 b): Intégration de la protection du patrimoine géologique dans l'aménagement du territoire en Suisse. Dans: Mém. Soc. géol. France, N. S., 165, 93-97. TEAM+, TÜSCHER URBANISME SA (1993): Plan sectoriel des paysages et des sites. Pré-étude. Fribourg, OCAT.

TEAM+, TÜSCHER URBANISME SA (1995): Plan sectoriel des paysages et des sites. Dossier d'avant-projet. Fribourg, OCAT.

TEAM+, TÜSCHER URBANISME SA (1996): Données de base pour le plan directeur cantonal. Diagnostic paysager dans le canton de Fribourg. Dans: OFEFP: Conception Paysage Suisse, Partie III Modules cantonaux. Berne.

WIEDEMBEIN, F. W. (1994): German developments in earth science conservation. Dans: Mém. Soc. géol. France, N. S., 165, 119-128.

WILSON, C. (Ed.) (1994): Earth Heritage Conservation. London, Geological Society \& The Open University. 